medgen 2019.31:320

https://doi.org/10.1007/s11825-019-00256-5

Online publiziert: 25 . Oktober 2019

(c) Deutsche Gesellschaft für Humangenetik e.V. 2019

glh
Deutsche Gesellschaft für Humangenetik e.V.

\section{GfH-Stellungnahme zum Rekontaktieren von Patienten}

Im Zeitalter von Genomik und Next Generation Sequencing hat die Frage des Rekontaktierens von Patienten bei der Neubewertung von genetischen Befunden an Bedeutung gewonnen. Diese stellt sich sowohl, wenn Primärbefunde, als auch wenn Sekundär- oder Zusatzbefunde neu bewertet werden. Dies kann Varianten in bereits zuvor ausgewerteten Genen oder Varianten in neu untersuchten Genen betreffen. Anlässe hierfür ergeben sich z. B. durch die Neubewertung von genetischen Varianten in Publikationen bzw. Datenbanken, durch die Identifikation neuer Krankheitsgene oder die Neuinterpretation in der Vergangenheit erhobener Befunde bei Anfragen von Familienangehörigen für eine prädiktive Testung.

ASHG, ESHG und ACMG haben zu diesem Thema aktuelle Stellungnahmen publiziert, die sich auf den wissenschaftlichen oder diagnostischen Kontext oder beides beziehen [1-3]. Diese Stellungnahmen behandeln die verschiedenen Aspekte des Rekontaktierens von Patienten umfassend. Im Kern sehen sie eine Pflicht zum Rekontaktieren, wenn eine neue Einschätzung des Befundes für die Gesundheit eines Patienten oder seine Lebensplanung von wesentlicher Bedeutung ist, stellen diese aber in ein realistisches Verhältnis zu vorhandenen Ressourcen. Darüber hinaus übertragen sie dem Patienten eine größere Verantwortung, in einem definierten Abstand aktiv nachzufragen, ob sich neue Erkenntnisse zu einem vorherigen Befund ergeben haben. Es wird keine Pflicht zum systematischen Re-Analysieren von Befunden von Seiten des betreuenden Arztes bzw. des Labors gesehen.

Da die genannten Stellungnahmen alle wesentlichen Aspekte des Rekontaktierens von Patienten umfassend abhandeln, ist die Kommission der Meinung, dass eine systematische eigene Abhandlung des Themas nicht erforderlich ist. Die Kommission schließt sich besonders den prägnanten Empfehlungen des ACMG [3] an.

Die Kommission sieht allerdings die Notwendigkeit, dass der Frage, wann und wie Patienten zu rekontaktieren sind, prospektiv mehr Bedeutung beigemessen wird. Sie spricht sich dafür aus, dass a. die Patienteninformation bei Forschungsvorhaben klare Angaben zur Frage des Rekontaktierens enthalten soll. Diese soll den Rahmen des durch die gegebenen Forschungsmittel Machbaren berücksichtigen. Der Patient soll die Möglichkeit haben, ein Rekontaktieren zu verweigern (opt-out).

b. bei der Mitteilung von unklaren oder unauffälligen Befunden diagnostischer Untersuchungen immer auf die Möglichkeit einer zukünftigen Neubewertung durch Änderung der Datenlage hingewiesen werden soll, die für den Patienten potenziell von Bedeutung sein kann. Zu diesem Zeitpunkt sollte geklärt werden, ob der Patient rekontaktiert werden möchte, und es sollten die unter c. genannten Punkte besprochen werden. c. mehr Verantwortung beim Patienten als primärem Verwalter seiner Gesundheitsdaten selbst liegen soll. Der Patient wird aufgefordert, sich bei unauffälligen oder unklaren Befunden nach einem zu definierenden bzw. im Befund genannten Zeitraum bei dem anfordernden Arzt oder dem durchführenden Labor, ggf. nach Besuch einer humangenetischen Einrichtung, hinsichtlich einer Aktualisierung des Befundes $\mathrm{zu}$ erkundigen. Es wird empfohlen, standardmäßig eine Kopie des Originalbefundes an den Patienten auszuhändigen. Der Patient soll zudem gebeten werden, dem Arzt, der die Befundmitteilung vornimmt, eventuelle Änderungen seiner Kontaktdaten mitzuteilen.

\section{Autoren:}

\section{Mitglieder der GfH-Kommission für Grundpositionen und Ethische Fragen}

Prof. Dr. Dr. med. Ute Moog, Heidelberg (Sprecherin)

Prof. Dr. Stefan Aretz, Bonn

Dr. med. Martin Kehrer, Tübingen

Prof. Dr. med. Christian Netzer, Köln

Dr. med. Rixa Woitschach, Hamburg

Prof. Dr. med. Dr. rer. nat. Birgit Zirn, Stuttgart

\section{Für den GfH-Vorstand}

Prof. Dr. med. Brigitte Schlegelberger

Prof. Dr. rer. nat. Thomas Eggermann

Prof. Dr. med. Olaf Rieß

Prof. Dr. med. Ute Hehr

Prof. Dr. rer. nat. Uwe Kornak

\section{Literatur}

1. Bombard $Y$, Brothers $K B$, Fitzgerald-Butt $S$, Garrison $N A$, Jamal $L$, James $C A$, Jarvik GP, McCormick JB, Nelson TN, Ormond KE, Rehm HL, Richer J, Souzeau E, Vassy JL, Wagner JK, Levy HP. The Responsibility to Recontact Research Participants after Reinterpretation of Genetic and Genomic Research Results. Am J Hum Genet 2019;104:578-595. PMID: 30951675

2. Carrieri D, Howard HC, Benjamin C, Clarke AJ, Dheensa S, Doheny S, Hawkins N, Halbersma-Konings TF, Jackson L, Kayserili H, Kelly SE, Lucassen AM, Mendes Á, Rial-Sebbag E, Stefánsdótttir V, Turnpenny PD, van EI CG, van Langen IM, Cornel MC, Forzano F; European Society of Human Genetics. Recontacting patients in clinical genetics services: recommendations of the European Society of Human Genetics. Eur J Hum Genet 2019;27:169-182. PMID: 30310124

3. David KL, Best RG, Brenman LM, Bush L, Deignan JL, Flannery D, Hoffman JD, Holm I, Miller DT, O'Leary J, Pyeritz RE; ACMG Social Ethical Legal Issues Committee. Patient re-contact after revision of genomic test results: points to consider a statement of the American College of Medical Genetics and Genomics (ACMG). Genet Med 2019;21:769-771. PMID: 30578420 